\title{
Reflection-Symmetric Second-Order Topological Insulators and Superconductors
}

\author{
Josias Langbehn, Yang Peng, ${ }^{*}$ Luka Trifunovic, Felix von Oppen, and Piet W. Brouwer \\ Dahlem Center for Complex Quantum Systems and Physics Department, Freie Universität Berlin, \\ Arnimallee 14, 14195 Berlin, Germany
}

(Received 12 August 2017; published 11 December 2017)

\begin{abstract}
Second-order topological insulators are crystalline insulators with a gapped bulk and gapped crystalline boundaries, but with topologically protected gapless states at the intersection of two boundaries. Without further spatial symmetries, five of the ten Altland-Zirnbauer symmetry classes allow for the existence of such second-order topological insulators in two and three dimensions. We show that reflection symmetry can be employed to systematically generate examples of second-order topological insulators and superconductors, although the topologically protected states at corners (in two dimensions) or at crystal edges (in three dimensions) continue to exist if reflection symmetry is broken. A three-dimensional secondorder topological insulator with broken time-reversal symmetry shows a Hall conductance quantized in units of $e^{2} / h$.
\end{abstract}

DOI: 10.1103/PhysRevLett.119.246401

Introduction.-After the discovery of topological insulators and superconductors and their classification for the ten Altland-Zirnbauer symmetry classes [1-3], the concept of nontrivial topological band structures has been extended to materials in which the crystal structure is essential for the protection of topological phases [4]. This includes weak topological insulators [5], which rely on the discrete translation symmetry of the crystal lattice, and topological crystalline insulators [6], for which other crystal symmetries are invoked to protect a topological phase. Whereas the original strong topological insulators always have topologically protected boundary states, weak topological insulators or topological crystalline insulators have protected boundary states for selected surfaces or edges only.

In a recent publication, Schindler et al. [7] proposed another extension of the topological insulator (TI) family: a higher-order topological insulator. Being crystalline insulators, these have well-defined faces and well-defined edges or corners at the intersections between the faces. An $n$th order topological insulator has topologically protected gapless states at the intersection of $n$ crystal faces, but is gapped otherwise [7]. For example, a second-order topological insulator in two dimensions $(d=2)$ has zero-energy states at corners, but a gapped bulk and no gapless edge states. Earlier examples of higher-order topological insulators and superconductors avant la lettre appeared in works by Benalcazar et al. [8-10] (see also Refs. [11,12]), who considered insulators and superconductors with protected corner states in $d=2$ and $d=3$ [13]. Sitte et al. showed that a three-dimensional topological insulator in a magnetic field of generic direction also acquires the characteristics of a second-order topological Chern insulator, with chiral states moving along the sample edges [14]. (see also [15] in the context of topological superfluid.)
Since a second-order TI has a topologically trivial $d$-dimensional bulk, from a topological point of view its boundaries are essentially stand-alone $(d-1)$-dimensional insulators, so that topologically protected states at corners (for $d=2$ ) or edges (for $d=3$ ) arise naturally as "domain walls" at the intersection of two boundaries if these are in different topological classes $[1,16,17]$. Similarly, the classification of $n$th order TIs derives from that of TIs in $d+1-n$ dimensions, i.e., the same classification of codimension $n$ topological defects [18] (see Ref. [19] for a scattering-approach based classification of $n$th order TIs). Note that, unlike for strong topological insulators and superconductors, which have protected states at all boundaries, $n$ th-order topological insulators and superconductors have topologically protected states at the intersection of $n$ boundaries only if (some of) these boundaries are in different topological classes; they do not necessarily have protected states at all intersections of $n$ boundaries.

Apart from their role in stabilizing well-defined crystal faces, crystalline symmetries are not required for the protection of higher-order TIs. However, crystal symmetries can be a key to ensure that a natural surface termination-, i.e., a surface termination that respects the crystal symmetries - automatically leads to a nontrivial higher-order topological phase. For example, Benalcazar et al. employed a combination of multiple reflection symmetries [9], whereas Schindler et al. considered $C_{4} \mathcal{T}$ symmetry, the product of a $\pi / 2$ rotation and time reversal, as well as a model with reflection symmetry [7].

In this Letter, we show that a single mirror symmetry is sufficient to construct models for second-order topological insulators and superconductors in $d=2$ and $d=3$ for all five Altland-Zirnbauer classes for which second-order topological insulators are allowed. Reflection-symmetric topological crystalline insulators were the first to be 

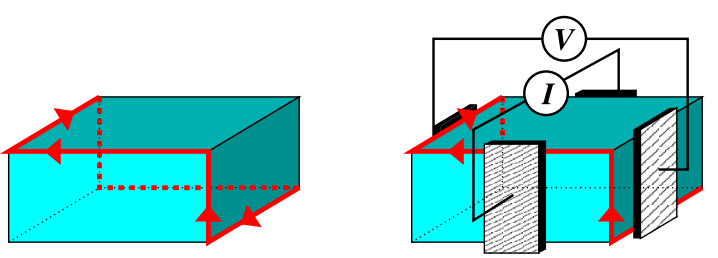

FIG. 1. A generic reflection-symmetric second-order topological insulator has chiral edge states winding around the crystal (left). If current and voltage contacts cover at most two neighboring edges, such a sample shows edge transport and, consequently, a quantized Hall effect in three dimensions (right).

realized experimentally $[17,35,36]$. A complete classification of reflection-symmetric topological insulators and superconductors exists for all ten Altland-Zirnbauer classes [20-22,32], and our construction makes use of this classification. Since the second-order topological phase does not require the reflection symmetry for its existence-see our general remarks above and our detailed discussion below-, in practice an approximate reflection symmetry may well be sufficient, which significantly enhances the prospects of an experimental realization.

An (approximately) reflection-symmetric threedimensional second-order topological insulator with broken time-reversal symmetry has chiral edge states winding around the sample if none of its crystal faces is reflection symmetric; see Fig. 1(a). Despite being three-dimensional, such crystals show a Hall effect with Hall conductance quantized in units of $e^{2} / h$, if current and voltage probes are attached such that they touch a single sample edge or two neighboring edges; see Fig. 1(b) [14]. Such a quantized Hall effect for a three-dimensional crystal is different from the "three-dimensional quantized Hall effect," which involves a topologically nontrivial bulk state and a quantized Hall conductivity [37-41]. Similarly, a reflection- and time-reversal-symmetric three-dimensional second-order topological insulator has a one-dimensional helical edge state winding around the crystal, corresponding to a quantized spin Hall effect in three dimensions. The experimental detection of such a quantized (spin) Hall effect should be an unambiguous experimental signature of a second-order TI.

Second-order topological insulators with reflecton symmetry.-Since corners and edges follow the classification of one-dimensional and two-dimensional topological insulators and superconductors, second-order topological insulators with protected zero-energy corner states or with gapless edge states can exist for selected Altland-Zirnbauer classes only; see Table I. We now provide a systematic method to construct examples of second-order topological insulators in all five nontrivial Altland-Zirnbauer classes, using a single reflection symmetry $\mathcal{R}$ to ensure the presence of topologically protected corner or edge states.

For each allowed Altland-Zirnbauer class, the construction requires (i) one or more pairs of crystal faces that are
TABLE I. The ten Altland-Zirnbauer classes are defined according to the presence or absence of time-reversal $(\mathcal{T})$, particle-hole $(\mathcal{P})$, and chiral symmetry $(\mathcal{C})$. A nonzero entry indicates the square of the antiunitary symmetry operations $\mathcal{T}$ or $\mathcal{P}$. Two-dimensional and three-dimensional reflection-symmetric topological crystalline phases that can be used for the construction of second-order topological insulator or superconductor phases are listed in the right two columns, together with the corresponding topological classification. The symbols $\mathcal{R}_{\sigma_{\mathcal{T}}}, \mathcal{R}_{\sigma_{\mathcal{P}}}$, $\mathcal{R}_{\sigma_{\mathcal{C}}}$, and $\mathcal{R}_{\sigma_{\mathcal{T}}, \sigma_{\mathcal{P}}}$ refer to a reflection operator that squares to one and commutes $(\sigma=+)$ or anticommutes $(\sigma=-)$ with $\mathcal{T}, \mathcal{P}$, or $\mathcal{C}$.

\begin{tabular}{|c|c|c|c|c|c|c|c|}
\hline Cartan & $\mathcal{T}$ & $\mathcal{P}$ & $\mathcal{C}$ & & $d=2$ & & $d=3$ \\
\hline A & 0 & 0 & 0 & 0 & $\ldots$ & $\mathbb{Z}$ & $\mathcal{R}$ \\
\hline AIII & 0 & 0 & 1 & $\mathbb{Z}$ & $\mathcal{R}_{+}$ & 0 & $\cdots$ \\
\hline AI & 1 & 0 & 0 & 0 & $\ldots$ & 0 & $\cdots$ \\
\hline BDI & 1 & 1 & 1 & $\mathbb{Z}$ & $\mathcal{R}_{++}$ & 0 & $\ldots$ \\
\hline $\mathrm{D}$ & 0 & 1 & 0 & $\mathbb{Z}_{2}$ & $\mathcal{R}_{+}$ & $\mathbb{Z}$ & $\mathcal{R}_{+}$ \\
\hline DIII & -1 & 1 & 1 & $\mathbb{Z}_{2}$ & $\mathcal{R}_{++}, \mathcal{R}_{--} \mathcal{R}_{-+}$ & $\mathbb{Z}_{2}$ & $\mathcal{R}_{++}, \mathcal{R}_{-+}$ \\
\hline AII & -1 & 0 & 0 & 0 & $\ldots$ & $\mathbb{Z}_{2}$ & $\mathcal{R}_{+}, \mathcal{R}_{-}$ \\
\hline CII & -1 & -1 & 1 & $\mathbb{Z}$ & $\mathcal{R}_{++}, \mathcal{R}_{--}$ & 0 & $\ldots$ \\
\hline $\mathrm{C}$ & 0 & -1 & 0 & 0 & $\ldots$ & $\mathbb{Z}$ & $\mathcal{R}_{+}, \mathcal{R}_{-}$ \\
\hline $\mathrm{CI}$ & 1 & -1 & 1 & 0 & $\cdots$ & 0 & $\ldots$ \\
\hline
\end{tabular}

mapped onto each other by reflection and (ii) a reflectionsymmetric topological crystalline phase that becomes trivial if the reflection symmetry is broken. The nontrivial topology of the corresponding Altland-Zirnbauer class in $d-1$ dimensions guarantees that the reflection-symmetrybreaking mass term that gaps out any boundary states existing in the presence of reflection symmetry is unique. Since this mass term must be odd under reflection, the two surfaces listed under (i) must be in different topological classes, ensuring the existence of zero energy (gapless) states at least two corners (edges). Table I lists the reflection-symmetric phases that meet these criteria. We emphasize again that the reflection symmetry is used to construct the second-order topological insulator; it is itself not essential for the existence of zero-energy corner states (for $d=2$ ) or gapless edge states (for $d=3$ ). The corner states (edge states) are robust against a reflection-symmetry breaking perturbation, as long as the bulk and edge (surface) gaps are not closed [42].

Below we discuss three examples in detail: A twodimensional second-order topological superconductor with Majorana corner states (class $D$ ), a three-dimensional second-order topological insulator with chiral edge states (class $A$ ), and a three-dimensional second-order topological insulator with helical edge states (class AII). In all cases we take reflection to map the momentum component $k_{1}$ into $-k_{1}$, leaving the other momentum components unchanged.

Second-order topological superconductor: Class D.For a superconductor with broken time-reversal and spinrotation symmetry, particle-hole symmetry $\mathcal{P}$ is the only relevant symmetry operation. Without loss of generality we may represent $\mathcal{P}$ by complex conjugation $K$ by working in 
a Majorana basis and the reflection operation by the Pauli matrix $\sigma_{1}$ in an orbital subspace, so that the Hamiltonian $H\left(k_{1}, k_{2}\right)$ satisfies

$$
H\left(k_{1}, k_{2}\right)=-H^{*}\left(-k_{1},-k_{2}\right)=\sigma_{1} H\left(-k_{1}, k_{2}\right) \sigma_{1} .
$$

Without reflection symmetry, class $D$ in two dimensions has a $\mathbb{Z}$ classification, where the integer topological number counts the number of chiral Majorana edge modes [43,44]. Chiral Majorana modes are incompatible with reflection symmetry. Instead, with reflection symmetry a $\mathbb{Z}_{2}$ topological structure remains $[20,21,32]$, counting the parity of the number of helical (i.e., counterpropagating) Majorana edge modes. A minimal reflection-symmetric nontrivial gapless edge state at a reflection-symmetric edge has edge Hamiltonian $H_{\text {edge }}=v k_{1} \sigma_{3}$, with $v$ the velocity, which is a trivial edge in the absence of $\mathcal{R}$. Indeed, upon breaking reflection symmetry, $H_{\text {edge }}$ is gapped out by a unique mass term $m \sigma_{2}$. An explicit model realizing this scenario is given by the four-band tight-binding Hamiltonian,

$$
\begin{aligned}
H= & \left(M-\cos k_{1}-\cos k_{2}\right) \tau_{2}+\tau_{1} \sigma_{3} \sin k_{1}+\tau_{3} \sin k_{2} \\
& +\lambda \tau_{2} \sigma_{1},
\end{aligned}
$$

with $0<|M|<2$ and $\lambda$ numerically small. The physical implementaion would require stacking two $p_{x} \pm i p_{y}$ superconductors [45] with opposite chirality and coupling them in such a way to gap out edge modes at nonreflection symmetric edges.

Computing the spectrum of low-lying excitations for a rectangular crystal with edges at $45^{\circ}$ with respect to the symmetry axis we find a zero-energy state well separated from higher-lying excitations by a gap. The wave function of the zero-energy states is localized near the two sample corners where the reflection-related edges meet, as shown in Figs. 2(a) and 2(b) for two different arrangements of the reflection line with respect to the corner of the crystal. The localized zero mode persists if the crystal is rotated, such that there are no longer any reflection-related edges [Fig. 2(c)].

Second-order topological insulator in three dimensions: Class A.-In three dimensions the presence of reflection symmetry allows for a topological crystalline phase with an
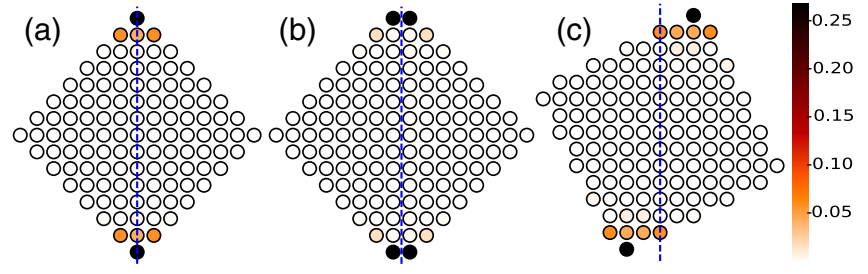

FIG. 2. Weight of the zero-energy wave function for the Hamiltonian (2) with $M=0.9$ and $\lambda=-0.25$ for three orientations of the crystal lattice. The reflection line is shown dashed. integer "mirror Chern number" enumerating gapless surface states at reflection-symmetric surfaces $[20,21,32,46]$. Using $\sigma_{1}$ to represent the reflection operation, such surface states have Hamiltonian $H_{\text {surface }}=v_{1} k_{1} \sigma_{3}+v_{2} k_{2} \sigma_{1}$. The unique mass term gapping out such surface states is $m \sigma_{2}$, which is odd under reflection. Explicitly, one may consider the fourband Hamiltonian

$$
\begin{aligned}
H= & \left(M-\cos k_{1}-\cos k_{2}-\cos k_{3}\right) \tau_{2} \sigma_{1} \\
& +\sigma_{3} \sin k_{1}+\tau_{1} \sigma_{1} \sin k_{2}+\tau_{3} \sigma_{1} \sin k_{3}+B \tau_{2},
\end{aligned}
$$

where $\sigma$ and $\tau$ are Pauli matrices in the space spanned by the unit-cell orbitals, two of which are even (odd) under reflection. For $1<M<3$ and $B$ numerically small this Hamiltonian describes a three-dimensional topologial insulator with a reflection-symmetric time-reversal symmetrybreaking term. To see how the bulk $B \tau_{2}$ term gives rise to the $\sigma_{2}$ term at the nonreflection symmetric facet, we first note that the details of the boundary do not have influence of the choice of the $B$ term. Thus to obtain facet Hamiltonian for one of the $y z$ facets it is sufficient to use low-energy expansion of the Hamiltonian (3) and model the boundary by the mass $M$ domain wall along the $x$ direction. This way we immediately conclude that the projector onto the $y z$-facet Hamiltonian is given by $\tau_{2} \sigma_{2}= \pm 1$; therefore, the term $B \tau_{2}$ acts like $\sim \sigma_{2}$ within the surface subspace (the proportionality factor does depend on the interface details).

Figure $3(\mathrm{~b})$ shows the band structure of a rectangular crystal with surfaces in the (110) and (110) direction, and
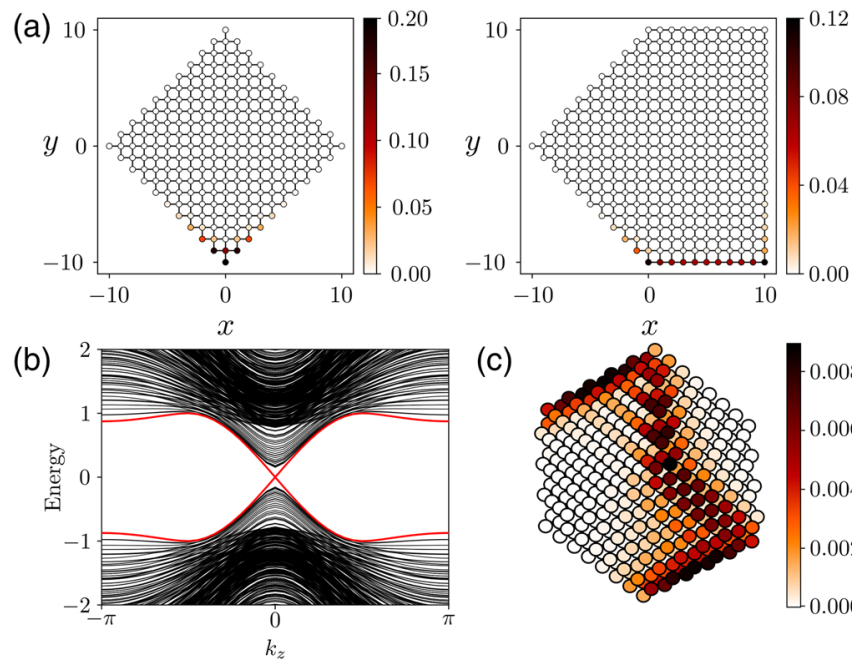

(c)

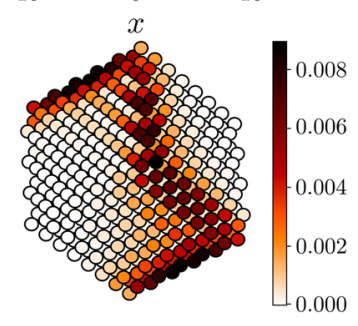

FIG. 3. (a) The crystal shape and the spatial profile of one of the in-gap states. Left: when the two surfaces meet at the reflection plane under a sharp angle, the in-gap state is well localized; right: one surface is perpendicular to the reflection plane, the in-gap state becomes completely delocalized on that surface. (b) Band structure of a rectangular crystal with periodic boundary conditions in the $k_{3}$ direction, for the model (3) with parameters $M=2, B=0.2$. (c) Weight of the zero-energy chiral edge states for a finite lattice with generic orientation with respect to the reflection plane. 
periodic boundary conditions in the $k_{3}$ direction. The two gapless chiral modes running in the positive and negative $k_{3}$ direction are located at the intersection of the (110) and (110) surfaces related by $\mathcal{R}$; see Fig. 3(a). We verified that the chiral edge states persist if the crystal orientation is rotated by an angle less than $45^{\circ}$ and migrates to the other pair of edges for larger rotation angles. Figure 3(c) shows the support of a chiral edge state for the above model Hamiltonian for a cubic sample randomly oriented with respect to the reflection plane (so that none of the facets are reflection symmetric).

Second-order topological insulator in three dimensions: class AII.-We represent the time-reversal and reflection operations by $\sigma_{2} K$ and $\sigma_{1}$, respectively, so that $H\left(k_{1}, k_{2}, k_{3}\right)$ satisfies

$$
\begin{aligned}
H\left(k_{1}, k_{2}, k_{3}\right) & =\sigma_{2} H\left(-k_{1},-k_{2},-k_{3}\right)^{*} \sigma_{2} \\
& =\sigma_{1} H\left(-k_{1}, k_{2}, k_{3}\right) \sigma_{1} .
\end{aligned}
$$

Hamiltonians with this symmetry have a $\mathbb{Z}$ topological classification $[20,21,32,46]$ which enumerates the number of surface states with Dirac-like dispersion $H_{\text {surface }}=$ $v_{1} k_{1} \sigma_{3}+v_{2} k_{2} \sigma_{1}$ at reflection-symmetric surfaces. The unique $\mathcal{R}$-breaking mass term that gaps out such a pair of surface states is $m \sigma_{2} \tau_{2}$, where $\tau_{2}$ is an additional Pauli matrix. (A single surface Dirac cone is protected by timereversal symmetry.) Since this mass term is odd under reflection, we expect an integer number of one-dimensional helical states at the intersection of two surfaces related by $\mathcal{R}$. An even number of helical states is unstable, however, to a local perturbation at the edge and can be gapped out without closing the gaps in the sample bulk or at the surfaces, consistent with the $\mathbb{Z}_{2}$ classification in Table $I$. (At this point our classification differs from that of Ref. [7], which does not allow for reflection-symmetry breaking perturbations at the crystal edge, thus arriving at a $\mathbb{Z}$ [22] classification.) As a specific example, we consider the eight-band Hamiltonian (a)

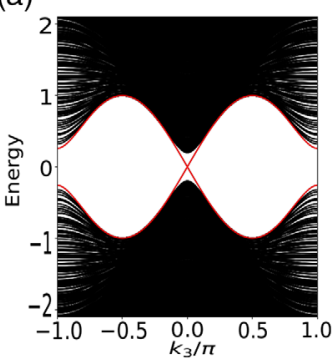

(b)

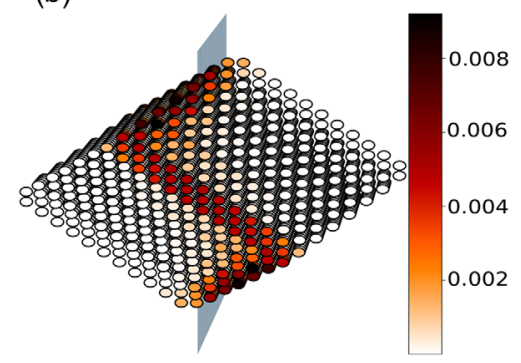

FIG. 4. (a) Band structure of a rectangular crystal with periodic boundary conditions in the $k_{3}$ direction, for the model (5) with parameters $M=2, B=0.8$. The crystal shape is the same as in Fig. 3. (b) Weight of the zero-energy chiral edge states for a finite lattice with generic orientation with respect to the reflection plane.

$$
\begin{aligned}
H= & {\left[\left(M-\cos k_{1}-\cos k_{2}-\cos k_{3}\right) \tau_{2} \sigma_{1}\right.} \\
& \left.+\sigma_{3} \sin k_{1}+\tau_{1} \sigma_{1} \sin k_{2}+\tau_{3} \sigma_{1} \sin k_{3}\right] \rho_{0}+B \tau_{2} \rho_{2},
\end{aligned}
$$

where $\rho_{0,1,2}$ are an additional set of Pauli matrices, $1<M<3$, and $B$ numerically small. One-dimensional band structure and weights for zero-energy states are shown in Fig. 4 for the same geometry as in the previous example.

Conclusion.-Although second-order topological insulators and superconductors can exist without a topological crystalline bulk phase, the existence of (approximate) crystalline symmetries can help in the construction of models or in the identification of materials that realize these phases. For reflection-symmetric topological crystalline insulators, the published literature has focused on reflection-symmetric surfaces, because these surfaces harbor topologically protected surface states $[4,17,35,36,46]$. We have shown that there is a good reason to look at crystals with arbitrarily oriented surfaces, because such crystals are good candidates for second-order TIs. Whereas the surface states of reflection-symmetric topological crystalline insulators are vulnerable to even weak perturbations that break the reflection symmetry, the associated edge states are robust and persist as long as surface and bulk gaps remain open. Combined with the unique prospect of isolated Majorana bound states (for two-dimensional second-order topological superconductors) or onedimensional chiral modes and a quantized Hall effect (for three-dimensional second-order TIs), higher-order TIs are a promising addition to the topological materials family. Very recently, some early experimental realizations of second-order topological insulators appeared [47-50], where Ref. [47] uses the bismuth nanowire which was previously shown to support edge states [51].

This work was motivated by a colloquium on C4Tsymmetric higher-order topological insulators by Titus Neupert at FU Berlin and subsequent discussions. We also thank Andrei Bernevig and Max Geier for discussions. We gratefully acknowledge support by projects $\mathrm{A} 03$ and $\mathrm{C} 02$ of the CRC-TR 183 and by the priority programme SPP 1666 of the German Science Foundation (DFG).

Note added.-Recently we became aware of related works [10,52].

*Corresponding author. yang.peng@ fu-berlin.de

[1] M.Z. Hasan and C. L. Kane, Rev. Mod. Phys. 82, 3045 (2010).

[2] B. A. Bernevig and T. L. Hughes, Topological Insulators and Topological Superconductors (Princeton University Press, Princeton, 2013).

[3] X.-L. Qi and S.-C. Zhang, Rev. Mod. Phys. 83, 1057 (2011). 
[4] C.-K. Chiu, J. C. Y. Teo, A. P. Schnyder, and S. Ryu, Rev. Mod. Phys. 88, 035005 (2016).

[5] L. Fu, C. L. Kane, and E. J. Mele, Phys. Rev. Lett. 98, 106803 (2007).

[6] L. Fu, Phys. Rev. Lett. 106, 106802 (2011).

[7] F. Schindler, A. M. Cook, M. G. Vergniory, Z. Wang, S. S. Parkin, B. A. Bernevig, and T. Neupert, arXiv:1708.03636.

[8] W. A. Benalcazar, J. C. Y. Teo, and T. L. Hughes, Phys. Rev. B 89, 224503 (2014).

[9] W. A. Benalcazar, B. A. Bernevig, and T. L. Hughes, Science 357, 61 (2017).

[10] W. A. Benalcazar, B. A. Bernevig, and T. L. Hughes, Phys. Rev. B 96, 245115 (2017).

[11] Y. Peng, Y. Bao, and F. von Oppen, Phys. Rev. B 95, 235143 (2017).

[12] F. Zhang, C. L. Kane, and E. J. Mele, Phys. Rev. Lett. 110, 046404 (2013).

[13] Reference [8] views the corner of a three-dimensional lattice as a disclination of the surface. In contrast, the corner states of a two-dimensional second-order TI exist as the corners of a "flat" surface.

[14] M. Sitte, A. Rosch, E. Altman, and L. Fritz, Phys. Rev. Lett. 108, 126807 (2012).

[15] G. E. Volovik, JETP Lett. 91, 201 (2010).

[16] R. Jackiw and C. Rebbi, Phys. Rev. D 13, 3398 (1976).

[17] T. H. Hsieh, H. Lin, J. Liu, W. Duan, A. Bansil, and L. Fu, Nat. Commun. 3, 982 (2012).

[18] J. C. Y. Teo and C. L. Kane, Phys. Rev. B 82, 115120 (2010).

[19] See Supplemental Material at http://link.aps.org/ supplemental/10.1103/PhysRevLett.119.246401 for a scattering-approach based classification of $n$th order TIs, which includes Refs. [9,16,20-34].

[20] C.-K. Chiu, H. Yao, and S. Ryu, Phys. Rev. B 88, 075142 (2013).

[21] K. Shiozaki and M. Sato, Phys. Rev. B 90, 165114 (2014).

[22] L. Trifunovic and P. W. Brouwer, arXiv:1707.06306.

[23] S. H. Simon, Phys. Rev. B 61, R16327 (2000).

[24] D. Meidan, T. Micklitz, and P. W. Brouwer, Phys. Rev. B 82, 161303 (2010).

[25] D. Meidan, T. Micklitz, and P. W. Brouwer, Phys. Rev. B 84, 195410 (2011).

[26] I. C. Fulga, F. Hassler, A. R. Akhmerov, and C. W. J. Beenakker, Phys. Rev. B 83, 155429 (2011).

[27] I. C. Fulga, F. Hassler, and A. R. Akhmerov, Phys. Rev. B 85, 165409 (2012).

[28] L. Fu and C. L. Kane, Phys. Rev. B 74, 195312 (2006).

[29] F. Merz and J. T. Chalker, Phys. Rev. B 65, 054425 (2002).
[30] A. R. Akhmerov, J. P. Dahlhaus, F. Hassler, M. Wimmer, and C. W. J. Beenakker, Phys. Rev. Lett. 106, 057001 (2011).

[31] A. Lau, J. van den Brink, and C. Ortix, Phys. Rev. B 94, 165164 (2016).

[32] T. Morimoto and A. Furusaki, Phys. Rev. B 88, 125129 (2013).

[33] T. Morimoto and A. Furusaki, Phys. Rev. B 88, 125129 (2013).

[34] R. B. Laughlin, Phys. Rev. B 23, 5632 (1981).

[35] Y. Tanaka, Z. Ren, T. Sato, K. Nakayama, S. Souma, T. Takahashi, K. Segawa, and Y. Ando, Nat. Phys. 8, 800 (2012).

[36] S.-Y. Xu et al., Nat. Commun. 3, 1192 (2012).

[37] B. I. Halperin, Jpn. J. Appl. Phys. 26, 1913 (1987).

[38] G. Montambaux and M. Kohmoto, Phys. Rev. B 41, 11417 (1990).

[39] J. T. Chalker and A. Dohmen, Phys. Rev. Lett. 75, 4496 (1995).

[40] M. Koshino, H. Aoki, K. Kuroki, S. Kagoshima, and T. Osada, Phys. Rev. Lett. 86, 1062 (2001).

[41] M. Koshino, H. Aoki, T. Osada, K. Kuroki, and S. Kagoshima, Phys. Rev. B 65, 045310 (2002).

[42] In principle, since the corners (edges) featured in the above construction may themselves be reflection symmetric, the reflection symmetry may allow for additional states. These, however, can be gapped out by any weak perturbation that breaks the reflection symmetry.

[43] G. E. Volovik, Zh. Eksp. Teor. Fiz. 94, 123 (1988) [Sov. Phys. JETP 67, 1804 (1988)].

[44] N. Read and D. Green, Phys. Rev. B 61, 10267 (2000).

[45] P. A. Lee, arXiv:0907.2681.

[46] J. C. Y. Teo, L. Fu, and C. L. Kane, Phys. Rev. B 78, 045426 (2008).

[47] A. Murani, A. Kasumov, S. Sengupta, Y. A. Kasumov, V. T. Volkov, I. I. Khodos, F. Brisset, R. Delagrange, A. Chepelianskii, R. Deblock, H. Bouchiat, and S. Guéron, Nat. Commun. 8, 15941 (2017).

[48] M. Serra-Garcia, V. Peri, R. Süsstrunk, O. R. Bilal, T. Larsen, L. G. Villanueva, and S. D. Huber, arXiv:1708.05015.

[49] C. W. Peterson, W. A. Benalcazar, T. L. Hughes, and G. Bahl, arXiv:1710.03231.

[50] S. Imhof, C. Berger, F. Bayer, J. Brehm, L. Molenkamp, T. Kiessling, F. Schindler, C. H. Lee, M. Greiter, T. Neupert, and R. Thomale, arXiv:1708.03647.

[51] O. Deb, A. Soori, and D. Sen, J. Phys. Condens. Matter 26, 315009 (2014).

[52] Z. Song, Z. Fang, and C. Fang, following Letter, Phys. Rev. Lett. 119, 246402 (2017). 